\title{
L'estimation de la vulnérabilité urbaine, un outil pour la gestion du risque
}

Approche à partir du cas de l'agglomération lyonnaise ${ }^{1}$

\author{
Samuel Rufat \\ Agrégé de géographie, doctorant \\ Géophile, UMR 5600
}

\begin{abstract}
Résumé : Le renforcement de la législation sur la gestion du risque, suite à la « loi Bachelot », conduit les approches par la seule exposition aux aléas à aggraver la situation des habitants. L'obligation de déplacer les équipements et les services publics hors des quartiers les plus exposés s'ajoute aux nuisances dues aux sources de danger. Une approche par la vulnérabilité pose des problèmes de définition des seuils, qui sont résolus par le recours aux analyses multivariées. A partir du cas lyonnais, l'utilisation de classifications automatiques est affinée pour obtenir une typologie de la vulnérabilité des îlots urbains. La vulnérabilité est cartographiée et superposée à une carte des aléas pour produire une carte synthétique du risque urbain. Elle permet de déplacer les préoccupations de la gestion du risque des espaces les plus exposés, survalorisés par la législation, aux espaces les plus vulnérables.
\end{abstract}

Mots clés: risque, vulnérabilité, classification automatique, cartographie, législation, gestion du risque.

\begin{abstract}
The reinforcement of risk management legislation, following the "Bachelot law", leads approaches based solely on hazard exposure to increase penalties on inhabitants. The obligation to move equipment and public services away from the most exposed neighbourhoods adds to the nuisances due to the sources of danger. An approach based upon vulnerability raises problems of definition of thresholds, which are solved through a multi-variant analysis. Based upon the case of Lyon, the use of automatic classifications is refined in order to obtain a typology of vulnerability of urban clusters. Vulnerability is mapped and superimposed to a hazard map in order to produce a synthetic map of urban risk. It allows shifting focus of the risk management from the most exposed areas, overrated by legislation, to the most vulnerable areas.
\end{abstract}

Key words: risk, vulnerability, automatic classification, cartography, legislation, risk management.

Les nuisances et dangers liés à l'industrie chimique et pétrochimique sont sensibles dans l'agglomération lyonnaise, en particulier depuis leur implantation massive dans les années 1960. Les catastrophes, comme celle des explosions en série des sphères de stockage d'hydrocarbures de la raffinerie de Feyzin le 4 janvier 1966, conduisent à la remise en cause de la localisation de l'industrie en ville. Les réponses politiques interviennent souvent après de telles catastrophes (Tchernobyl, Toulouse...), elles sont la plupart du temps d'ordre réglementaire et ne parviennent pas à trancher entre le déplacement des activités à l'origine du danger et celui des populations qui vivent à proximité, deux solutions qui ont des coûts importants. Dans cette perspective, la gestion du risque se centre sur la définition des zones d'exposition aux aléas et sur la maîtrise du foncier dans un objectif de protection de la population. Cette démarche fonctionnelle conduit à déplacer les activités, les équipements et les services publics en dehors des quartiers les plus exposés aux aléas et donc à en défavoriser les habitants déjà pénalisés par la proximité des sources de danger.

\section{La gestion du risque technologique à Lyon}

- Une exposition multiple aux aléas

L'agglomération lyonnaise est en partie exposée aux aléas hydrologiques, géologiques ou technologiques et parfois à plusieurs d'entre eux. Ces derniers donnent lieu

\footnotetext{
${ }^{1}$ Cette estimation a été élaborée pour la réalisation d'un Atlas des risques technologiques, en collaboration avec l'Agence d'urbanisme de Lyon, dont l'auteur tient à remercier ici toute l'équipe, en particulier Olivier Roussel et Marion Boissel Chagnard.
} 
à des accidents industriels et à des accidents de transport de matières dangereuses ${ }^{2}$, regroupés selon deux types de scénarios: incendie et explosion (flux thermique et surpression), fuite accidentelle (rupture de confinement et concentration de toxicité grave). Les aléas biologiques et nucléaires sont liés au laboratoire P4 dans le $7^{\text {ème }}$ arrondissement de Lyon et aux centrales du Bugey, de Saint Alban et de Creys-Malville, qui se situent entre 30 et $50 \mathrm{~km}$ du centre ville. Ils ont une très faible probabilité d'occurrence, mais en cas d'accident une vaste étendue du territoire serait touchée. Ils ne font pas l'objet de mesures de maîtrise de l'urbanisation. Dans une perspective de gestion, les périmètres d'exposition retenus sont ceux pris en compte dans les documents réglementaires ${ }^{3}$.

Une étude rétrospective sur les catastrophes au cours du $X X^{\text {ème }}$ siècle a été publiée en octobre 2000 par le Grand Lyon. Elle montre que les accidents liés aux aléas technologiques se sont multipliés après 1960. Ils ont les impacts les plus importants en termes de dégâts et de coûts, même si la catastrophe la plus meurtrière reste l'éboulement de la colline de Fourvière en 1930 (39 morts). Leur gravité est renforcée par les " effets domino ", à la fois effet de site, comme à Feyzin en 1966, et effet de substance, quand différents produits interagissent, produisant soit une explosion, soit un nuage toxique, comme lors de l'incendie du Port Edouard Herriot en août 1990. Les deux approches, prospective et rétrospective, font ressortir l'axe Saône Rhône, à la fois du fait de la superposition des périmètres d'exposition à différents aléas et de la concentration des stocks et des flux de matières dangereuses (possibilité "d'effet domino »). Cet axe est également le premier concerné par le renforcement du cadre légal.

- Un cadre légal renforcé, la loi Bachelot

Après la catastrophe de l'usine AZF à Toulouse le 21 septembre 2001, la loi du 30 juillet 2003, dite «loi Bachelot ", relative à la prévention des risques et à la réparation des dommages, vise à garantir une meilleure acceptabilité et renforcer la maîtrise du risque. Elle modifie l'évaluation des aléas technologiques en introduisant dans l'étude de danger la prise " en compte de la probabilité d'occurrence, la cinétique (dynamique dans le temps) et la gravité des accidents potentiels » (art. 4). L'étude de danger reste à la charge de l'exploitant lors de la demande d'autorisation en préfecture. Alors qu'en France comme en Europe (directives Seveso), la gestion du risque se contentait de ne pas aggraver les situations en s'appuyant sur le gel du foncier, la «loi Bachelot» prévoit également de remédier aux situations déjà graves par la « reconquête » des périmètres exposés. Cette "reconquête » s'effectue par expropriation, droit de délaissement et de préemption, selon la gravité de l'exposition, avec la mise en place de Plans de Prévention des Risques Technologiques (PPRT) avant le 31 juillet 2008.

\section{- Lyon face à l'obligation légale de « reconquête »}

Les aléas liés à la production, au stockage et aux flux de transport générés par l'activité industrielle ont fait l'objet de nouvelles études de danger. La DRIRE (Direction régionale de l'industrie de la recherche et de l'environnement ${ }^{4}$ ) les a utilisés pour établir les périmètres d'exposition, que la Préfecture a ensuite validés ${ }^{5}$. La spatialisation des aléas repose sur le calcul des seuils des conséquences, qu'il s'agisse de concentrations de produits toxiques, de flux thermiques, ou de surpressions. Les périmètres sont encore délimités selon l'approche "déterministe », ce sont des cercles autour des installations dont le rayon correspond à la distance maximale (en fonction des vents) pour un seuil donné de conséquence (ZIMMERMANN E., 1996). Les dernières études de danger retenaient deux seuils :

- Z1, zone létale pour au moins $1 \%$ des personnes présentes ;

\footnotetext{
${ }^{2}$ Une matière dangereuses est une substance qui, par ses propriétés physiques ou chimiques, peut présenter un danger grave pour l'homme, les biens, l'environnement. On distingue en général ces substances selon leur nature explosive, inflammable, toxique, radioactive, ou corrosive.

${ }^{3}$ Les Plans de Surfaces Submersibles pour les aléas hydrologiques, les plans du BRGM pour les aléas géologiques, les Porter à Connaissance de la Préfecture pour les aléas industriels, les axes routiers (DDE), ferroviaires (SNCF) et fluviaux (VNF) autorisés aux flux de matières dangereuses, ainsi que les conduites souterraines (Préfecture).

${ }^{4}$ www.rhone-alpes.drire.gouv.fr/

5 Porter à Connaissance de décembre 2003, complété en août 2004 pour le Grand Lyon et périmètres de Givors et Grigny, 2003, les maires ont eu connaissances des nouvelles cartes à partir de décembre 2002.
} 
- Z2, zone d'apparition d'effets irréversibles ou de blessures graves ;

Malgré des efforts de confinement et la cessation d'une partie des activités, ces périmètres sont identiques à ceux des scénarios de référence de la DRIRE de 1990. Ils sont plus étendus que les anciennes zones de protection, donnant lieu à une maîtrise de l'urbanisation, qui ne dépassaient pas 500 mètres autour des sites et dont la surface s'était réduite lors des révisions successives des POS (DONZE J., 1996 et 2001). Leur inscription dans les documents d'urbanisme concernait moins de 15000 personnes et 2000 écoliers et ne donnait lieu qu'à un gel du foncier. Les nouveaux périmètres concernent près de 90000 personnes, 60000 emplois et 20000 écoliers, collégiens et lycéens, soit 7 à $10 \%$ de l'agglomération lyonnaise (tableau).

Exposition aux aléas technologiques dans l'agglomération lyonnaise

\begin{tabular}{|c|c|c|c|c|c|c|}
\hline & Population & $\begin{array}{c}\text { Résidences } \\
\text { Principales }\end{array}$ & Emplois & $\begin{array}{c}\text { Enseignemen } \\
\text { t primaire }\end{array}$ & $\begin{array}{c}\text { Enseignemen } \\
\text { t secondaire }\end{array}$ & $\begin{array}{c}\text { Enseignemen } \\
\text { t supérieur }\end{array}$ \\
\hline Z1 & $\begin{array}{c}18000 \\
(1,5 \%)\end{array}$ & $\begin{array}{c}\text { environ } \\
7800\end{array}$ & $\begin{array}{c}16050 \\
(2,6 \%)\end{array}$ & $\begin{array}{c}1550 \\
(1,2 \%)\end{array}$ & $\begin{array}{c}800 \\
(0,6 \%)\end{array}$ & 0 \\
\hline Z2 & $\begin{array}{c}47800 \\
(3,9 \%)\end{array}$ & $\begin{array}{c}\text { environ } \\
21000\end{array}$ & $\begin{array}{c}37100 \\
(6 \%)\end{array}$ & $\begin{array}{c}6100 \\
(4,7 \%)\end{array}$ & $\begin{array}{c}7250 \\
(5,4 \%)\end{array}$ & $\begin{array}{c}1650 \\
(1,5 \%)\end{array}$ \\
\hline $\begin{array}{c}\text { périmètres } \\
\text { Givors/Grigny }\end{array}$ & $\begin{array}{c}21250 \\
(1,7 \%)\end{array}$ & $\begin{array}{c}\text { environ } \\
\mathbf{9} 250\end{array}$ & $\begin{array}{c}4350 \\
(0,7 \%)\end{array}$ & $\begin{array}{c}1350 \\
(1 \%)\end{array}$ & $\begin{array}{c}1600 \\
(1,2 \%)\end{array}$ & 0 \\
\hline $\begin{array}{c}\text { TOTAL } \\
\text { exposés }\end{array}$ & $\begin{array}{c}\mathbf{8 7} \mathbf{0 5 0} \\
\mathbf{( 7 \% )}\end{array}$ & $\begin{array}{c}\text { environ } \\
\mathbf{3 8 ~ 0 0 0}\end{array}$ & $\begin{array}{c}\mathbf{5 7 5 0 0} \\
\mathbf{( 9 , 4} \%)\end{array}$ & $\begin{array}{c}\mathbf{8} \mathbf{9 5 0} \\
\mathbf{( 6 , 9} \%)\end{array}$ & $\begin{array}{c}\mathbf{9} \mathbf{6 5 0} \\
\mathbf{( 7 , 1} \%)\end{array}$ & $\begin{array}{c}\mathbf{1 6 5 0} \\
\mathbf{( 1 , 5} \%)\end{array}$ \\
\hline
\end{tabular}

\begin{tabular}{|c|c|c|c|c|c|c|}
\hline & $\begin{array}{l}\text { Hôpitaux et } \\
\text { Cliniques } \\
\text { (lits) }\end{array}$ & $\begin{array}{l}\text { Maisons de } \\
\text { Retraites }\end{array}$ & $\begin{array}{l}\text { Stades } \\
\text { (places) }\end{array}$ & $\begin{array}{l}\text { Piscines } \\
\text { (capacité) }\end{array}$ & $\begin{array}{c}\text { Cinémas } \\
\text { (places) }\end{array}$ & $\begin{array}{l}\text { Théâtres } \\
\text { (places) }\end{array}$ \\
\hline $\mathrm{Z1}$ & 0 & $50(0,4 \%)$ & $\begin{array}{c}1650 \\
(1,8 \%)\end{array}$ & $430(1,8 \%)$ & 395 (2 \%) & $685(2,3 \%)$ \\
\hline Z2 & $\begin{array}{c}1000 \\
(10,3 \%)\end{array}$ & 490 (4,3 \%) & $\begin{array}{l}44750 \\
(48 \%)\end{array}$ & 2870 (12\%) & 790 (4,1 \%) & $1430(4,9 \%)$ \\
\hline $\begin{array}{l}\text { périmètres } \\
\text { Givors/Grigny }\end{array}$ & 0 & $40(0,3 \%)$ & 950 (1 \%) & $530(2,2 \%)$ & 0 & $600(2 \%)$ \\
\hline $\begin{array}{l}\text { TOTAL } \\
\text { exposés }\end{array}$ & $\begin{array}{c}1000 \\
(10,3 \%)\end{array}$ & $\begin{array}{c}580 \\
(5,1 \%)\end{array}$ & 47650 (51\%) & $\begin{array}{c}3830 \\
(16 \%)\end{array}$ & $\begin{array}{c}1185 \\
(6,1 \%)\end{array}$ & $\begin{array}{c}2715 \\
(9,2 \%)\end{array}$ \\
\hline
\end{tabular}

La « reconquête » de ces périmètres exposés, d'abord délimités pour la mise à jour des plans d'intervention et non la maîtrise du foncier, est envisagée avec la mise en place des plans de prévention, mais elle pose de sérieux problèmes. D'abord en raison des équipements qui s'y trouvent, comme le principal hôpital, Lyon Sud à Pierre Bénite, ou le plus grand stade, à Gerland dans le $7^{\text {ème }}$ arrondissement de Lyon. Ce sont 5 à $50 \%$ des équipements recevant du public qui sont compris dans les zones d'atteintes irréversibles: leur coût, leur taille et leur rôle au sein de l'agglomération ne permettent pas d'envisager leur déplacement rapide. Ensuite, elles comprennent de nombreux logements sociaux (Feyzin, Givors et Saint Fons), dont la production augmente, mais elle insuffisante tant par rapport aux besoins qu'au regard des objectifs fixés par la loi SRU (déficit de 12500 logements locatifs sociaux en 2004). Enfin, les coûts générés seraient très lourds, avec d'importantes expropriations, alors que l'Etat n'a toujours pas précisé sa participation.

Les collectivités locales craignent que l'effort en faveur de la maîtrise du risque et de son acceptabilité ne repose que sur elles. La transcription mécanique des périmètres d'exposition dans les PPRT, envisagée au début 2005, conduit à définir l'importance des mesures selon la seule gravité maximale des conséquences, par exemple en déclarant

\footnotetext{
${ }^{6}$ Total sans double compte sur le périmètre du ScoT de l'agglomération lyonnaise (72 communes).
} 
l'expropriation d'utilité publique dans tous les périmètres d'effets mortels établis. Depuis les années 1990, la DRIRE avait privilégié l'autre solution, la diminution du risque à la source. Les municipalités redoutent le déplacement des activités ou le départ de certaines entreprises, qui sont parmi les principaux contributeurs à la taxe professionnelle, notamment Total $\left(1^{\mathrm{er}}\right)$, Arkéma et Rhodia. Pour sortir de cette impasse, la raffinerie de Feyzin a été nommée site pilote avec la mise en place d'un PPRT « expérimental ». La commune met en avant sa volonté de ne pas aggraver la situation des habitants du quartier le plus exposé (les Razes). Elle propose de renforcer la protection des bâtiments existants, de commander de nouvelles études de danger et d'adopter une approche plus nuancée de la réalisation des PPRT (SAUGE-GADOUD, 2005).

Le sud de l'agglomération lyonnaise voit son développement en grande partie bloqué par ce renforcement de la réglementation qui insiste sur l'exposition aux aléas, alors qu'une autre approche du risque est possible.

\section{La vulnérabilité urbaine, concept et méthodes}

La décennie 1990, que l'ONU a consacrée à la réduction des risques, a confirmé le passage du hazard paradigm, qui considère le risque comme une "altérité ", à la prise en compte du risque comme construction sociale, en insistant sur la vulnérabilité (PIGEON P., 2002, p. 457). La vulnérabilité s'exprime en résistance des systèmes exposés, et en résilience, capacité des systèmes à absorber le changement (DAUPHINE A., 2001, p. 17-20). La vulnérabilité est d'abord l'exposition physique, mais toujours potentielle, à un aléa, puis une certaine fragilité face à cet événement et enfin la méconnaissance des comportements à adopter en cas de catastrophe (VEYRET Y., 2003, p. 31). II existe un consensus pour séparer le risque de l'aléa, probabilité d'occurrence d'un phénomène dangereux. La définition du risque comme conjonction d'un aléa et d'une vulnérabilité est critiquée car fonctionnaliste, mais cette approche est la plus opératoire pour sa spatialisation (NOVEMBER V., 2000, p. 260). L'analyse des facteurs de vulnérabilité a donné lieu à différentes méthodes proposant des solutions pour réduire le risque et améliorer les réponses des sociétés (D’ERCOLE et al., 1994, p. 94).

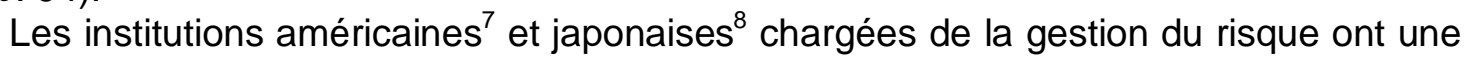
approche comptable de la vulnérabilité. Elle est analysée en terme de coût prévisible de l'exposition des personnes, infrastructures et biens aux aléas et de coût/bénéfice des mesures de protection. II s'agit d'une confusion entre l'exposition et la vulnérabilité, les personnes et infrastructures exposées sont définit comme des «enjeux ». Les institutions internationales (Banque mondiale, $\mathrm{ONU}^{9}$ ) analysent la vulnérabilité dans sa dimension sociale. Leurs études commencent par l'inventaire des aléas sur un territoire, puis analysent la fragilité des systèmes exposés (forte densité, engorgement des réseaux...), enfin leurs capacités d'adaptation et d'adoption de comportements opératoires en cas de crise. Mais les estimations formelles de la vulnérabilité reposent sur la définition de seuils et de coefficient arbitraires, que les développements soient par la suite algorithmiques ou matriciels.

Ainsi, dans l'ouvrage de synthèse publié par l'ONU sur le risque dans les métropoles, Sergio Puente propose une méthode d'analyse de la vulnérabilité urbaine (MITCHELL J. K., 1999, p. 295-334) qui repose sur une matrice multicritère. Elle permet de combiner des facteurs hétérogènes et donc de prendre en compte à la fois les facteurs de fragilité et de résilience des systèmes. L'analyse est présentée sous forme de matrice, avec les valeurs d'une trentaine d'indicateurs (âge, densité, revenu, matériaux de construction, réseau de gaz, sous-sol amplifiant les ondes sismiques...) auxquels est attribué un coefficient de renforcement de la vulnérabilité de 1 à 5 , à la discrétion de l'auteur. Cette méthode pose les

\footnotetext{
${ }^{7}$ Federal Emergency Management Agency, www.fema.gov ; US Department of Housing and Urban Development, www.hud.gov Fire and Disaster Management Agency, www.fdma.jp/en/; Ministry of Land, Infrastructures and Transport, www.mlit.go.jp/english/

${ }^{9}$ Voir les ouvrages cités, www.worldbank.org, et la Stratégie Internationale de l'ONU pour la prévention des catastrophes, www.unisdr.org
} 
bases d'une analyse de la vulnérabilité reposant sur les retours d'expérience ${ }^{10}$. Elle est cependant arbitraire dans la définition des seuils et l'attribution du degré de renforcement de la vulnérabilité. Le recours aux analyses multivariées permet de surmonter ces problèmes.

\section{Etapes de la mise au point de l'estimation de la vulnérabilité}

L'analyse est faite à l'échelle de l'îlot ${ }^{11}$ pour les 72 communes de l'agglomération lyonnaise ${ }^{12}$, soit 528 unités spatiales. La première étape, l'analyse des aléas selon les approches probabiliste et rétrospective, conduit à séparer les accidents en deux types: phénomènes rapides endommageant les infrastructures, touchant le bâti et les personnes (glissement de terrain rapide, surpression...) ; phénomènes lents, nécessitant une évacuation et/ou un confinement et touchant les populations qui sont à l'extérieur et/ou dans la zone d'exposition (montée des eaux, nuage toxique...). L'étape suivante consiste à choisir une première série d'indicateurs de vulnérabilité en fonction de ces accidents.

- Définition d'indicateurs de base, première typologie

La densité, qui est un indicateur de la concentration des habitants et des flux, est considérée comme le facteur le plus évident de vulnérabilité (BLANCHER Ph., 1996, p. 25). Mais elle est souvent aussi l'indicateur de la concentration de ressources, permettant une bonne réponse aux situations d'urgence. Du fait de son rôle ambigu, la densité ne sera prise en compte que dans les premières étapes de l'analyse. Pour un même aléa, les conséquences des catastrophes varient selon les catégories de personnes : les plus jeunes et les plus âgés sont à la fois plus fragiles et présentent plus de difficultés à être évacués (DAUPHINE A., 2001, p. 23-24). Les classes 0-9 et plus de 75 ans ont été retenues comme les plus cohérentes : prendre des classes plus larges en augmente le poids presque sans en modifier la distribution spatiale. Les personnes de mobilité réduite sont plus difficiles à évacuer et les personnes en habitat précaire (mobile ou de fortune) sont plus fragiles en cas d'incendie, de surpression ou de dispersion d'un nuage toxique. Au regard des accidents connus, cinq critères de base sont donc retenus: densité, population jeune (moins de 10 ans), population âgée (plus de 75 ans), population de mobilité réduite et population en habitat mobile ou de fortune. Ils peuvent tous être analysés à partir des données du dernier recensement général de la population (RGP 1999).

Une classification automatique sur ces premiers indicateurs permet de regrouper les 528 unités spatiales en cinq classes. Afin de localiser plus précisément les populations vulnérables, en particulier dans les communes périphériques où les îlots recouvrent les limites communales, la cartographie découpe dans les îlots les espaces non bâtis (IGN, BD Carto). Etant donné le nombre réduit de critères, un secret statistique pèse sur les 46 îlots les moins peuplés lors du recensement.

Carte 1 : Estimation de la vulnérabilité de l'agglomération lyonnaise sur 5 critères de base Rufat_Lyon_carte1

Cette première analyse permet de différencier les profils de forte vulnérabilité selon la composition de leur population (jeune ou âgée) mais présente des inconvénients. La prise en compte de la densité se traduit par un gradient centre périphérie : le profil le plus vulnérable se retrouve exclusivement dans le centre ville. Cette étape sert de base à une analyse approfondie, prenant en compte non seulement la vulnérabilité des personnes, mais aussi la vulnérabilité des territoires.

\section{- Elargissement des critères retenus à partir des retours d'expérience}

La vulnérabilité d'un territoire correspond à la fois à la vulnérabilité individuelle des personnes qui s'y trouvent, à la vulnérabilité de ses infrastructures et à la vulnérabilité

\footnotetext{
${ }^{10}$ La prise en considération des données recueillies lors du déroulement et des répercussions des accidents contemporains peut aboutir à une meilleure connaissance de leurs mécanismes, ces résultats sont exploités dans un but préventif.

${ }_{11}^{1}$ IRIS 2000, îlot de regroupement statistique de l'INSEE pour l'interprétation du dernier recensement général (RGP 1999).

12 En retenant le périmètre du SCOT de l'agglomération lyonnaise.
} 
" sociétale ", qui dépend de la répartition de la population, de sa réaction en cas de crise et de la capacité sociale de gestion de la crise.

L'expérience montre que les personnes en situation précaire ou marginale sont plus fragiles en cas de catastrophe et que leur capacité à surmonter la crise est réduite (VEYRET Y., 2003). L'analyse de la campagne de prévention en Rhône Alpes ${ }^{13}$ souligne que la sensibilisation de la population diminue au cours du temps, mais que son efficacité est croissante en fonction du niveau de formation. Les données sur le niveau de formation peuvent être extrapolées comme indiquant la plus ou moins grande connaissance des conduites à tenir en cas d'accident. Le niveau de formation a aussi été utilisé comme indicateur de la capacité à trouver de nouvelles réponses en situation de crise (KREIMER A., 2003, p. 213).

La vulnérabilité des infrastructures a été estimée par deux enquêtes, l'une sur l'habitat collectif en état dégradé, l'autre sur les capacités d'accueil des établissements recevant du public (données de l'Agence d'urbanisme, 2004-2005). Ces équipements ont été regroupés en fonction du type et de l'horaire de fréquentation en cinq catégories: santé (hôpitaux, cliniques et maisons de retraite où un lit correspond en général à trois personnes en tenant compte du personnel ainsi que des visites), sport (stades, piscines et salles de sport), culture (musées, théâtres, cinémas), administration et enseignement (écoles, collèges, lycées et supérieur), gares et hypermarchés. Les infrastructures liées aux activités dangereuses sont en partie intégrées dans l'analyse des aléas, elles n'ont pas été réutilisées pour éviter les doubles comptes. Par contre, les axes de transports, qui devraient être considérés pour leur rôle à la fois dans l'acheminement des secours et dans l'évacuation de la population, n'ont pas été pris en compte par manque de données (la DDE ne fait pas de comptage exhaustif des flux) et par manque de compatibilité avec les autres données, recueillies à l'échelle des îlots. Les forces de l'ordre et les pompiers (SDIS du Rhône) indiquent que l'ensemble des 72 communes est accessible en moins de 10 minutes, ce qui par ailleurs est un important facteur de réduction de la vulnérabilité.

Cet élargissement des critères conduit à retenir 38 variables. En écartant les variables redondantes (avec un taux de corrélation supérieur à 0,6 ), on peut alléger la part des variables socioéconomiques au profit des seuls nombre de chômeurs de plus d'un an (données RGP 1999), qui apparaît comme un indicateur synthétique de la précarité, et nombre des personnes de plus de 15 ans sans aucun diplôme (en dehors de celles qui sont en cours de formation, RGP 1999). De même, la plupart des variables sur les résidences principales sont écartées au profit de la population en habitat précaire (RGP 1999) et en habitat collectif dégradé (Agence d'urbanisme). Les fortes corrélations permettent une réduction à 13 variables sans une perte trop lourde d'information et en ménageant un équilibre dans le poids des différents groupes d'indicateurs. Deux séries de modifications ont été effectuées sur la prise en compte de la densité. D'une part, pour limiter l'influence des découpages administratifs, on a calculé la densité de la population dans les seuls espaces bâtis ; d'autre part, cette densité des espaces bâtis a été considérée comme variable témoin (illustrative), elle ne détermine plus la constitution des classes mais elle peut servir à l'interprétation de la typologie obtenue sans peser dans les calculs.

\section{- Analyse sur les 12 variables retenues, typologie et cartographie}

Une classification automatique sur les 12 variables retenues, plus la densité du bâti, permet de regrouper les 528 unités spatiales en cinq classes:

- Vulnérabilité très forte (profil 5) : la plus forte concentration des établissements recevant du public, à l'exception de ceux du type santé, avec une proportion de population de mobilité réduite, population en habitat précaire et une densité légèrement supérieures à la moyenne (58 unités), l'autocorrélation spatiale ${ }^{14}$ négative de cette classe montre une dispersion qui traduit les choix d'implantation de ces équipements ;

\footnotetext{
${ }^{13}$ Campagne d'information et de prévention des risques technologiques dans les périmètres d'évacuation en Rhône Alpes du printemps 2003, enquête d'impact de l'Institut Fournier en juin-juillet 2004.

4 Effet de voisinage entre unités spatiales, si cette corrélation est positive, c'est que les lieux voisins tendent à se ressembler, on observe des sous ensembles spatiaux homogènes, si elle est négative, les lieux voisins tendent à s'opposer.
} 
- Vulnérabilité forte, population jeune (profil 4) : la plus forte proportion de population jeune liée à une importante vulnérabilité socioéconomique avec les plus fortes proportions de chômeurs de plus d'un an, de population sans diplôme et en habitat collectif dégradé (130 unités), l'autocorrélation spatiale positive de cette classe reflète en partie la concentration des populations précaires dans l'Est et le Sud de l'agglomération ;

- Vulnérabilité forte, population âgée (profil 3) : la conjonction entre les plus fortes proportions des populations âgée et de mobilité réduite, avec les équipements de type santé, hôpitaux, cliniques et maisons de retraites (69 unités spatiales), l'autocorrélation spatiale négative de cette classe identifie la distribution des îlots accueillant à la fois une population et des équipements vulnérables ;

- Vulnérabilité moyenne (profil 2) : une population âgée importante avec presque tous les autres critères inférieurs à la moyenne, à l'exception de la densité (157 unités), l'autocorrélation spatiale positive de cette classe reflète en partie la concentration de la population âgée dans l'Ouest et le Nord de l'agglomération ;

- Vulnérabilité faible (profil 1) : dernière classe dans laquelle presque tous les critères sont largement inférieurs à la moyenne (100 unités) ;

- Le secret statistique pèse sur les 14 îlots regroupant moins de 30 résidences principales lors du recensement.

Carte 2 : Estimation de la vulnérabilité de l'agglomération lyonnaise, classification automatique sur 12 variables

Rufat_Lyon_carte2

L'intérêt d'avoir affiné l'analyse apparaît en comparant cette dernière étape avec les premiers résultats obtenus à partir des 5 critères de base. Elle permet d'abord de faire émerger une classe regroupant les fortes concentrations d'établissements recevant du public, qui est un facteur de vulnérabilité bien plus fort que la simple densité des îlots, dont nous avons vu qu'elle est un indicateur ambigu. Ensuite, le lien entre population âgée et population de mobilité réduite est précisé par la corrélation avec les équipements de type santé pour une partie des îlots. La concentration sur ces îlots d'une série de facteurs indique la vulnérabilité de ces populations, même si elles semblent bien encadrées. Enfin, elle relativise la vulnérabilité des îlots présentant une importante proportion de population âgée lorsqu'ils ne présentent pas d'autres facteurs de vulnérabilité.

Cependant, cette méthode est limitée par le type de données utilisées. Elles sont d'origine, de nature et de date différentes, surfaciques pour les données issues du recensement (IRIS 2000, RGP 1999), ponctuelles pour les enquêtes 2004-2005. Cette diversité peut être traitée par l'analyse multivariée, mais elle pose des problèmes d'interprétation en périphérie en raison de la grande taille des îlots. Ainsi, à Tassin la Demi Lune, l'îlot central est plus étendu que la zone dense et vulnérable concentrant d'importants équipements autour de la gare. Cette méthode pose également problème dans la hiérarchisation des classes obtenues par classification automatique. On a fait le choix de définir le profil liant une population jeune avec une vulnérabilité socioéconomique forte comme plus vulnérable que celui présentant une conjonction d'une population âgée, avec une mobilité réduite et des équipements de type santé. Ce choix repose sur le rôle ambivalent des équipements de type santé sur le renforcement de la vulnérabilité : ils sont un important facteur de vulnérabilité sociétale, mais aussi un vecteur d'encadrement facilitant une intervention rapide et une bonne prise en charge de la population.

\section{Superposition de la cartographie des aléas et de la vulnérabilité}

La carte est un outil indispensable à la gestion du risque, parce qu'elle permet de le matérialiser, de rendre visible l'invisible (PROPECK-ZIMMERMANN E., 2001). La définition du risque comme conjonction d'un aléa et de la vulnérabilité des systèmes exposés permet sa lecture directe par la superposition de deux cartographies. Mais la multiplication des 
informations liée à la superposition de tous les périmètres d'aléas (hydrologiques, géologiques, technologiques...) conduirait à faire cette carte à si grande échelle que cela morcellerait l'agglomération. Pour obtenir une carte permettant une lecture synthétique, on peut choisir de faire plusieurs cartes de l'ensemble de l'agglomération en fonction des types d'aléas, puis de les comparer.

Carte 3 : Risque dans l'agglomération lyonnaise, aléas industriels et estimation de la vulnérabilité Rufat_Lyon_carte3

La cartographie du risque technologique comprend alors une première superposition des aléas industriels et de la vulnérabilité, en prenant pour référence les périmètres retenus lors des dernières études de danger (août 2004). Une seconde superposition, celle des axes supportant des flux de matières dangereuses avec la vulnérabilité, est plus difficile car les flux ne peuvent donner lieu au même traitement que les stocks : il faut tenir compte à la fois des axes de circulation et du volume des flux. Les flux de matières dangereuses par voies ferrée et fluviale sont quantifiés par les autorités gestionnaires des infrastructures ou du fret (SNCF et RFF d'une part, VNF et Port E. Herriot d'autre part), ils peuvent être facilement cartographiés. Les flux de matières dangereuses par voie fluviale et ferroviaire sont importants (voir graphiques), mais la route en concentre encore près des deux tiers. Les données utilisées pour ces derniers sont des estimations de la DDE du Rhône pour l'année 2004.

Flux annuel de matières dangereuses en milliers de tonnes circulant par voie fluviale sur l'axe Saône Rhône au port Edouard Herriot (données VNF)

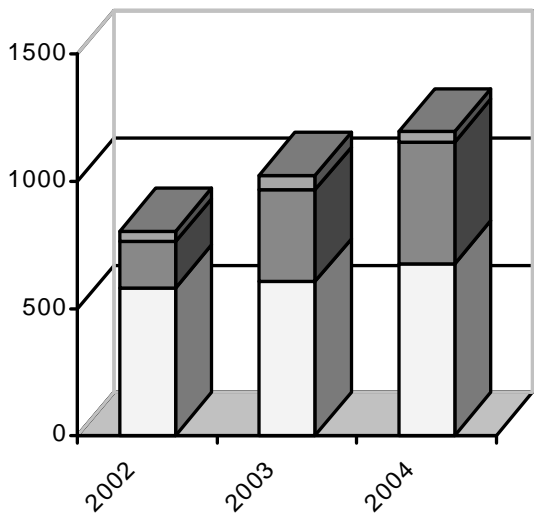

$\square$ Produits pétroliers $\square$ Produits chimiques $\square$ Engrais
Flux annuel de matières dangereuses en milliers de tonnes à la gare de la Part Dieu en 2004 (données SNCF) total : 4,85 millions de tonnes

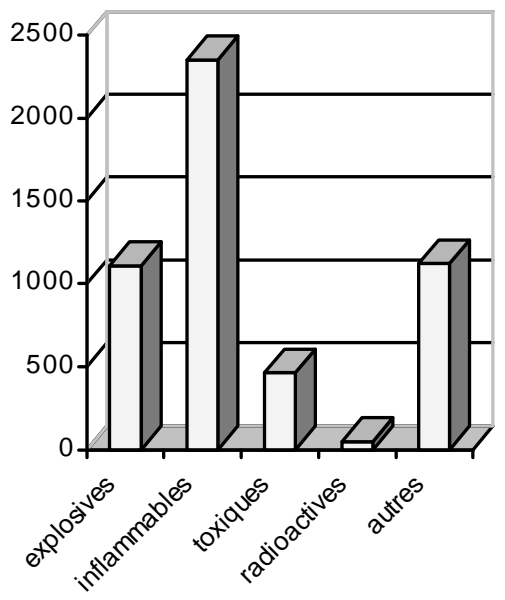

Les axes de transport supportant des flux de matières dangereuses ne donnent pas lieux à la mise en place de périmètres d'exposition, mais les conséquences d'un accident peuvent être presque aussi étendues que dans le cas des stockages. Ainsi, une fuite sur un wagon isoconteneur de 18 tonnes de chlore pourrait donner lieu à des conséquences mortelles sur un rayon de 750 à 1200 mètres, en fonction de la durée de la fuite et de la vitesse du vent (Porté à connaissance, août 2004). Le passage de flux importants de matières dangereuses, en majorité inflammables et explosives, mais également toxiques et radioactives, par le centre ville de l'agglomération conduit à l'exposition d'une partie importante de la population (près de 100000 personnes à moins de 100 mètres de ces flux).

Carte 4 : Risque dans l'agglomération lyonnaise, 


\section{axes de circulation des TMD et estimation de la vulnérabilité} Rufat_Lyon_carte4

La lecture de ces cartes conduit à revenir sur la définition de l'axe rhodanien comme source majeure de risque au sein de l'agglomération. II connaît une exposition simultanée à plusieurs aléas et accueille d'importants stocks ainsi que les flux de matières dangereuses. Mais la carte du risque lié aux stockages industriels montre que les secteurs les plus vulnérables ne sont pas tous exposés et que les périmètres d'exposition contiennent des îlots de profil peu vulnérable, ce qui est un important facteur de diminution du risque. En revanche, la carte du risque lié aux flux de matières dangereuses montre que presque tous les îlots de profil de vulnérabilité forte et très forte se trouvent à proximité des axes concernés (routiers, ferrés, fluviaux). Ce déplacement des enjeux pour la gestion du risque est particulièrement important à l'heure ou le renforcement législatif récent a conduit à survaloriser l'axe rhodanien.

\section{Estimation de la vulnérabilité et perspectives de gestion du risque}

Dans le cas des stockages industriels, les secteurs exposés de profil moins vulnérable peuvent soit présenter des facilités au vu de l'obligation légale de "reconquête » (pas d'équipements), soit permettre de justifier la priorité donnée aux ouvrages de protection sur l'expropriation. A Neuville sur Saône, la forte vulnérabilité à la marge du périmètre d'exposition est due à l'implantation d'une grande surface au bord de la zone industrielle. Mais la majeure partie des îlots de profil le plus vulnérable sont exposés par le seul scénario de rupture de la conduite aérienne de chlore sur le site d'Arkéma à Pierre Bénite. Cet accident pourrait générer un nuage toxique qui atteindrait non seulement l'hôpital Lyon Sud ( $10 \%$ des lits d'hôpital) et le complexe sportif de Gerland (48\% de la capacité des stades), mais aussi le centre de Pierre Bénite et l'ouest de Saint Fons qui sont du second profil le plus vulnérable (population jeune, vulnérabilité socioéconomique). Dans ce cas, l'importance des équipements et le nombre des logements concernés rend très difficile la « reconquête " et conduit à envisager le confinement total ou la cessation de l'activité dangereuse.

Plus généralement, les principaux périmètres sont définis autour des stockages de produits dérivés du fluor (HFA) de la chaîne du froid et du chlore dans les plastiques (PVC) auxquels est également liée une conduite souterraine qui traverse l'Est de l'agglomération. Une réduction des stockages, voire une réorientation de l'activité sur d'autres intrants, entraîne une réduction des périmètres dans le Nord de la Vallée de la Chimie, avec la fermeture du site de Butagaz par exemple. Ce mouvement accompagne les processus de reconversion, l'implantation d'entreprises de logistique et le développement des activités de recherche, avec la mise en place d'un "pôle de compétitivité », qui nécessite pourtant le maintien d'unités de production.

Dans le cas des flux de matières dangereuses, il n'existe pas de périmètre d'exposition. Mais les équipements recevant du public se trouvent à moins de 200 mètres des axes de circulation autorisés à ces flux, à l'exception des deux principaux hôpitaux (Lyon Sud à Pierre Bénite et Edouard Herriot dans le $8^{\text {ème }}$ arrondissement de Lyon). Les principaux facteurs de risque sont le périphérique et l'autoroute $A 7$, ces axes souvent saturés traversent de nombreux îlots des profils les plus vulnérables, ainsi que les gares voyageurs, surtout quand elles accueillent des espaces commerciaux (Part Dieu, Perrache) ou quand elles se trouvent dans des centres villes qui concentrent les équipements et les populations vulnérables (Genay, Givors, Tassin la Demi Lune). Des efforts pour augmenter le contournement de l'agglomération par le transit de matières dangereuses sont entrepris. Pour la route, le plan de circulation semble adapté, puisque 3500 personnes habitent à moins de 100 mètres du contournement Est (A46) contre plus de 20000 le long de l'axe A6/A7, mais la DDE du Rhône avoue que l'interdiction d'emprunter le tunnel de Fourvière n'est pas toujours respectée. Le centre de l'agglomération supporte la circulation de matières dangereuses par voie ferrée, qui traversent les gare de Perrache (plus de 17000 
montées/descentes par jour en 2004) et de la Part Dieu (plus de 13 000), alors qu'une très faible partie de ces produits y est destinée.

L'estimation de la vulnérabilité va au-delà de la transformation des périmètres d'exposition en glacis fonciers. Elle permet à la fois de déplacer les préoccupations des espaces les plus exposés aux espaces exposés les plus vulnérables, et une meilleure prise en compte des aléas dus aux flux traversant le centre ville.

\section{Limites}

Cependant, cette méthode appelle certaines remarques. Elle répond aux besoins de la gestion et de l'aide à la décision en alternant entre vision globale et analytique, mais cette approche conduit à des synthèses qui lui font parfois perdre en précision. La prise en compte simultanée de tous les aléas est efficace en terme de gestion, pour saisir les interactions possibles, mais la multiplication des informations rend leur superposition cartographique difficile : seule l'exposition aux aléas industriels et aux flux de matières dangereuse a été présentée. Les aléas biologiques et nucléaires n'ont pas été cartographiés du fait de l'exposition presque uniforme de l'ensemble de l'agglomération, ils ne sont pas assez pris en considération. Enfin, les espaces non bâtis ont été considérés comme homogènes et écartés de l'analyse.

L'utilisation de données issues du recensement donne le lieu d'habitation (résidence principale) comme cadre de référence. La prise en compte simultanée des emplois aurait généré un double compte d'une partie de la population. Les infrastructures de transport intègrent de même dans l'analyse des données qui ne sont pas toujours compatibles avec les îlots. Ces données sur les axes et les flux permettraient de faire des analyses différenciées selon les tranches horaires, en lien avec la typologie des équipements. Elles ne sont qu'en partie considérées, d'abord par l'analyse des différents flux de matières dangereuses, ensuite au travers de la fréquentation des gares, enfin par le rappel de la saturation de certains axes dans l'interprétation des cartes.

L'efficacité de la mise en carte dans l'approche fonctionnelle se fait au détriment de la prise en compte des jeux d'acteurs et des comportements en cas de crise, qui a fait l'objet d'une approximation. Elle reste également soumises à une part d'arbitraire dans la délimitation des périmètres d'exposition et des unités spatiales (COMBY J., 2004, p. 83). Un algorithme de lissage surmonte ces difficultés, mais il supprime les îlots qui sont nécessaires aux politiques de gestion.

\section{Conclusion}

La mise au point progressive de cette méthode permet de faire une estimation fiable de la vulnérabilité des îlots d'une grande agglomération exposée à différents aléas. Les cartes obtenues permettent une lecture synthétique de la vulnérabilité et du risque, elles matérialisent des informations nombreuses et complexes et sont un outil efficace tant pour l'aide à la décision que la sensibilisation ou l'évaluation des enjeux au-delà des limites communales. Mais elle reste perfectible, notamment en intégrant des données SIRENE et des données Corine Land Cover pour élargir l'analyse à tous les types d'espace de l'agglomération, ainsi qu'en trouvant une solution pour intégrer plus de données sur les axes et les flux. Le problème de la diversité des aléas pourrait être résolu par la création d'un indice d'aléa en fonction de l'éloignement des différentes sources, mais cela pose des problèmes de seuil très différents de ceux qui ont été abordés.

En conduisant à déplacer les enjeux majeurs des espaces les plus exposés (axe rhodanien) aux espaces les plus vulnérables exposés, la méthode présentée est un outil efficace de gestion du risque. Elle permet également une meilleure prise en compte du rôle des flux de matières dangereuses dans la création de situations à risque, en localisant précisément les problèmes de cœxistence entre des sources de danger (stocks ou flux) et des populations les plus vulnérables ou d'importants équipements recevant du public. 


\section{Références:}

Approche historique du risque. Mise en place d'un recensement sur les risques naturels et technologiques, 2000, Lyon, Agence d'urbanisme, octobre 2000, 2 vol.

Atlas des risques technologiques et de la vulnérabilité dans l'agglomération lyonnaise, 2005, Lyon, Agence d'urbanisme, Grand Lyon et Sepal, $46 \mathrm{p}$.

Blancher Ph., Paquiet P. et Zampa Ch., 1996, Industries chimiques et territoire : contraintes et opportunités de développement, Revue de Géographie de Lyon, vol. 71 n¹, p. 23-30

CERTU, 2003, Risque industriel et territoires en France et en Europe. Etat des lieux et perspectives, Lyon, Ministère de l'Equipement et des Transports, $123 \mathrm{p}$.

CombY J., 2004, La mise en carte et risques de dérives technocratiques dans la prévention des risques, Risques naturels et aménagement en Europe, Actes du colloque de La Défense d'octobre 2002, Paris, Armand Colin, p. 79-85

DAUPHINE A., 2001, Risques et catastrophes. Observer, spatialiser, comprendre, gérer, Paris, Armand Colin, $384 \mathrm{p}$.

D’ERCOLE R. et al., 1994, Les vulnérabilités des sociétés et des espaces urbanisés, Revue de Géographie Alpine, n 4, p. 87-96

DONZE J., 2005, «Les bassins du risque industriel : l'exemple de la vallée du Rhône», Géoconfluences, http://geoconfluences.ens-Ish.fr/doc/transv/Risque/RisqueScient3.htm

DONZE J., 2001, Risque technologique et urbanisation (Saint Fons, Pierre-Bénite, Salaise sur Sanne) Colloque risque et territoires, UMR 5600, pré actes tome 1, Lyon, Vaux en Velin.

DONZE J., 1996, L'impact des risques technologiques sur l'urbanisation. Les communes de Pont de Claix et de Saint Fons, Revue de Géographie de Lyon, vol. 71 n¹, p. 45-55

Kreimer A. (ed.), 2003, Building safer cities. The future of disaster risk, Washington, The World Bank, Disaster Risk Management series $n^{\circ}$ 3, $299 \mathrm{p}$.

MitCheLL J. M., 1999, Crucibles of hazard : mega cities and disasters in transition, Tokyo, UN University Press, $418 \mathrm{p}$.

NovemBer V., 2000, Les territoires du risque. Le risque comme objet de réflexion géographique, Bern, Peter Lang, $332 \mathrm{p}$.

PIGEON P., 2002, Réflexion sur les notions et les méthodes en géographie des risques dits naturels, Annales de Géographie, $\mathrm{n}^{\circ}$ 627-628, sept.-oct. 2002, p. 452-470

Sauge-Gadoud D., 2005, Feyzin, une ville de référence dans la maîtrise du risque technologique, Cahiers de l'IAURIF, n¹42, p. 194-203

VeYRET Y. (dir), 2003, Les Risques, Paris, Sedes, coll. « Diem », 255 p.

Propeck-Zimmermann E. et SaINT Gerand T., 2001, Modélisation cartographique des RTM : de la connaissance du risque à sa gestion ou objectiver le risque dans un SIG pour l'objectiviser, Colloque risque et territoires, UMR 5600, pré actes tome 1, Lyon, Vaux en Velin.

ZIMMERMANN E., 1996, De l'usage de la cartographie dans l'appréhension des risques technologiques majeurs, Revue de Géographie de Lyon, vol. 71, n¹, p. 11-16

Samuel RUFAT

ENS LSH - Géophile - UMR 5600

15 parvis René Descartes

69366 Lyon Cedex 07

tél. 0437376335

Samuel.Rufat@ens-Ish.fr 